\title{
THE COHOMOLOGY GROUPS OF A FIBRE SPACE WITH FIBRE A SPACE OF TYPE $\varkappa(\pi, n)$. II
}

\author{
BY \\ W. H. COCKCROFT
}

1. Introduction and statement of results. The problem of computing the cohomology groups of a fibre space whose fibre is of type $\Re(\pi, n)$, and whose base space and characteristic class are known, is one of the important problems in present day algebraic topology. There are two existing techniques which can be applied to the problem. The first of these is, of course, the technique of spectral sequences (see e.g. $\left(^{1}\right)[4]$ ). The second is the method of G. Hirsch [7]. This paper is concerned with the application of this latter method to the problem.

I shall consider, therefore, fibre spaces $(E, B, F)$ in the sense of J. P. Serre [9], with arcwise connected base space $B$, and fibre $F$ of type $\Re(\pi, n)$. The cohomology theory of fibre spaces used will be that of Serre, loc. cit., using singular cubes, and his notation will be used as far as is possible.

The following assumptions will be made. Firstly, coefficients will be assumed to be taken in a commutative ring $K$ with a unit element. Secondly, the fundamental group $\pi_{1}(B)$ will be assumed to operate trivially on the (unitary) cohomology $K$-module $H^{*}(F ; K)$ of the fibre, which will be assumed to have a homogeneous $K$-basis, finite in each dimension.

These conditions ensure that the first term, $E_{1}^{*}$, of the spectral sequence in cohomology associated with $(E, B, F)$ is isomorphic with the tensor product, (over $K), C^{*}(B ; K) \otimes_{K} H^{*}(F ; K)=C^{*}(B ; K) \otimes_{K} H^{*}(\pi, n ; K)$. They are certainly fulfilled $\left({ }^{2}\right)$ in case $\pi$ is a finitely generated abelian group, and $K=Z_{p}$, the ring of integers reduced modulo a prime $p$. A discussion of the possible relaxation of these conditions to include the case when $\pi$ is not finitely generated, i.e. when the $K$-basis of $H^{*}(F ; K)$ need not be finite in each dimension, will be included. The exposition, particularly in $\$ 2$ below, is, I think, made somewhat more readable by making the more stringent assumption (which seems also to be of more practical value).

Under these conditions, then, the method of G. Hirsch, loc. cit., gives a monomorphism $\left({ }^{3}\right)$

Received by the editors August 15, 1957.

(1) The determination of the groups $H^{q}\left(\pi, n ; Z_{p}\right)$ by H. Cartan [2], enables one to extend to higher dimensions the results of [4]. The interpretation of the transgression homomorphism used in [4], in terms of the cohomological operations corresponding to $H^{q}\left(\pi, n ; Z_{p}\right)$ for $q=n$, $n+1, n+2$, is replaced by the interpretation in terms of the general operations with coefficients in $Z_{p}$, e.g. the Steenrod powers (cf. e.g. $\$ 5$ below).

(2) See [2].

$\left({ }^{3}\right)$ As is noted in $\$ 2$ below, the method of Hirsch is not of course restricted to the case when $F$ is of type $\mathfrak{K}(\pi, n)$. 


$$
u: C^{*}(B ; K) \otimes_{K} H^{*}(\pi, n ; K) \rightarrow C^{*}(E ; K)
$$

such that the image $\boldsymbol{u}\left(C^{*}(B ; K) \otimes_{K} H^{*}(\pi, n ; K)\right)$ is a graded sub-K-module of $C^{*}(E ; K)$, stable under the coboundary operator $d: C^{*}(E ; K) \rightarrow C^{*}(E ; K)$. By means of $\boldsymbol{u}$ the operator $d$ can be "pulled back" to $C^{*}(B ; K) \otimes_{K} H^{*}(\pi, n ; K)$ and a coboundary operator $\partial$ can be obtained in this tensor product, such that the cohomology modules given by $\partial$ (with the usual (total) grading of the tensor product) are isomorphic with the corresponding cohomology modules $H^{r}(E ; K)$, for all $r$. Stated in precise terms in the present context, we have as follows the theorem of Hirsch.

Theorem of Hirsch. For all integers $m \geqq 0$ there exists a coboundary operator.

$$
\partial: \sum_{p+q=m} C^{p}(B ; K) \otimes_{K} H^{q}(\pi, n ; K) \rightarrow \sum_{p+q=m+1} C^{p}(B ; K) \otimes_{K} H^{q}(\pi, n ; K)
$$

such that the cohomology modules $H^{m}\left(C^{*}(B ; K) \otimes_{K} H^{*}(\pi, n ; K)\right)$ which are defined by this operator are isomorphic with the cohomology modules $H^{m}(E ; K)$.

Now let $\partial_{B}: C^{p}(B ; K) \rightarrow C^{p+1}(B ; K)$ denote the coboundary operator in $B$. Further, recall that given any element, and therefore in particular any basis element, say $\mathrm{x} \in H^{q}(\pi, n ; K), 0<q<2 n$, there exists $\mathrm{y} \in H^{q+1}(\pi, n+1 ; K)$ such that $\mathrm{x}=\sigma^{*} \mathrm{y}$ where $\sigma^{*}: H^{q+1}(\pi, n+1 ; K) \rightarrow H^{q}(\pi, n ; K)$ denotes the (cohomology) suspension isomorphism in these dimensions. Let

$$
T(y): H^{n+1}(B ; \pi) \rightarrow H^{q+1}(B ; K)
$$

denote the cohomological operation associated with $y$, and if $\boldsymbol{k}^{n+1} \in H^{n+1}(B ; \pi)$ denotes the characteristic cohomology class of $(E, B, F)$, let $T(y) k^{n+1}$ denote a chosen cocycle in the class $T(\boldsymbol{y}) \boldsymbol{k}^{n+1}$. In dimensions $0<m<2 n$ the Hirsch coboundary operator can now be made explicit, in these terms, as follows.

Theorem. In case $0<m<2 n$ the coboundary operator $\partial$ of the Theorem of Hirsch can be defined by setting

$$
\begin{aligned}
& \partial(b \otimes 1)=\partial_{B} b \otimes 1, \\
& \partial(b \otimes x)=\partial_{B} b \otimes x-(-1)^{p}\left\{b \cup T(y) k^{n+1}\right\} \otimes 1,
\end{aligned}
$$

for all $b \in C^{p}(B ; K)$, and all basis elements $x \in H^{q}(\pi, n ; K)$, where $p+q=m$, $\sigma^{*} y=x$, and 1 denotes the unit element in $H^{*}(\pi, n ; K)$.

This theorem will be referred to as the main result of the paper. Its object, evidently, is to make explicit in dimensions $0<m<2 n$ the group $H^{m}\left(C^{*}(B ; K)\right.$ $\left.\otimes H^{*}(\pi, n ; K)\right)$ given by the Hirsch method. The theorem is proved in $\S 3$ below. In fact the arrangement of the rest of the paper in detail is as follows.

First, in $\$ 2$, there is given for the convenience of the reader a reconstruction of the proof of the Theorem of Hirsch within the framework of the Serre cubical singular cohomology of fibre spaces. This section closes with 
two brief notes. The first outlines what is known about how the conditions, under which the Hirsch Theorem has been proved, can be relaxed. This covers, in particular, the case when $\pi$ is not finitely generated. The subsequent required alterations in the proof as given are briefly indicated. The second note deals with the analogous theorem in homology. Next, in $\S 3$, it is shown that since the fibre $F$ of the space $(E, B, F)$, which is to be considered, is of type $\mathcal{K}(\pi, n)$, the coboundary operator $\partial$ which is given by the Hirsch method can be expressed up to dimensions $2 n-1$ (i.e. within the "suspension range") in terms of the transgression in the cohomology spectral sequence associated with $E$. The theory of cohomological operations then allows this fact to be exploited and the proof of the main result is completed, making explicit the Hirsch coboundary operator up to dimension $2 n-1$. Again this section closes with a brief note on the nature of the coboundary operator when $\pi$ is not finitely generated. In $\S 4$, results of the first paper under this title [4] are reobtained in strengthened form as an illustration of the power of the method. In particular a generalization of a group construction of Eilenberg-Maclane [5, Theorem II] is obtained. In the final section, §5, there is given a brief comparison, from the purely practical point of view, of the two methods of computing the cohomology groups of $E$, namely the Hirsch method as given here, and the spectral sequence method as used, e.g., in [4]. It appears that in any practical computations one would be driven back to methods analogous to those of [4], whichever method is used.

The paper is independent of [4] except in $\$ 4$ and $\S 5$ where reference is made to the results and spectral sequence methods of [4]. Since these latter methods are standard ones, I have not hesitated in $\$ 4$ and $\$ 5$ to accept their use and implications, without explicitly carrying out computations analogous to those of $[4]$.

2. The method of Hirsch. I recall first, for convenience, the various facts arising from the thesis of Serre [9] which will be required later. As far as is possible I use his notation. It should be noted that throughout this section the fibre $F$, of the fibre spaces $(E, B, F)$ which are considered, need not be of type $\mathcal{K}(\pi, n)$.

S1. First, then, in calculating the terms $E_{1}^{*}$ and $E_{2}^{*}$ of the cohomology spectral sequence associated with a fibre space $(E, B, F)$ Serre constructs a monomorphism into the term $E_{0}^{*}$ of the spectral sequence, namely

$$
\phi^{*}: C^{*}\left(B ; C^{*}(F ; K)\right) \rightarrow E_{0}^{*},
$$

with left inverse $\psi^{*}$. Both $\phi^{*}$ and $\psi^{*}$ are cochain mappings in the sense that they commute with the coboundary operator of $C^{*}(F ; K)$ in $C^{*}\left(B ; C^{*}(F ; K)\right)$, and with the coboundary operator $d_{0}^{*}: E_{0}^{*_{p}} \rightarrow E_{0}^{*_{p}}$ of the spectral sequence. Thus each induces homomorphisms of the respective cohomology groups obtained from these coboundary operators. In fact, if

$$
C^{*}(E ; K)=A^{*}=A^{* 0} \supset A^{* 1} \supset \cdots \supset A^{* p} \supset A^{* p+1} \supset \cdots
$$


is the Serre filtration of $C^{*}(E ; K)$ and if $Z^{*}(F ; K)$ and $Z^{*}\left(A^{* p} / A^{* p+1}\right)$ denote respectively the $K$-module of cocycles of $F$ with coefficients in $K$, and the $K$-module of $d_{0}^{*}$-cocycles of $A^{* p} / A^{* p+1}=E_{0}^{* p}$, then there is a commutative diagram

$$
\begin{gathered}
C^{p}\left(B ; Z^{*}(F ; K)\right) \underset{\psi^{*}}{\stackrel{\phi^{*}}{\rightleftarrows}} Z^{*}\left(A^{*_{p}} / A^{* p+1}\right) \\
\lambda \downarrow \\
C^{p}\left(B ; H^{*}(F ; K)\right) \underset{\phi^{* *}}{\stackrel{\psi^{* *}}{\leftrightarrows} H^{*}\left(A^{* p} / A^{* p+1}\right)=E_{1}^{* p}}
\end{gathered}
$$

where $\lambda, \mu$ denote the natural homomorphisms $\left({ }^{4}\right)$ mapping cocycles into their respective cohomology classes, and $\phi^{* *}, \psi^{* *}$ are the homomorphisms induced by $\phi^{*}, \psi^{*}$ respectively. As of course is proved in [9], $\phi^{* *}$ is an isomorphism onto $H^{*}\left(A^{* p} / A^{* p+1}\right)$, with inverse $\psi^{* *}$.

S2. Secondly, the natural homomorphisms $i^{*}: C^{*}(E ; K) \rightarrow C^{*}(F ; K)$, and $p^{*}: C^{*}(B ; K) \rightarrow C^{*}(E ; K)$, which are induced respectively by the injection mapping $i: F \rightarrow E$ and the projection mapping $p: E \rightarrow B$ of the fibre space, can be interpreted within the context of the spectral sequence ${ }^{(5)}$. Thus let $C_{r}^{*_{p}}$ denote as usual the elements of $A^{* p} C A^{*}=C^{*}(E ; K)$ which have coboundaries in $A^{* p+r}$, and let $\left.{ }^{4}\right) \beta: C_{r}^{*}{ }^{*} \rightarrow Z^{*}\left(A^{* p} / A^{* p+r}\right)$ denote the natural epimorphism arising therefrom, for any $p, r$. Then, in particular, there are commutative diagrams

$$
Z^{*}(F ; K)=C^{0}\left(B ; Z^{*}(F ; K)\right) \stackrel{\psi^{*}}{\longleftarrow} Z^{*}\left(\left.\right|_{\uparrow} ^{\left.A^{*} / A^{* 1}\right)}\right.
$$

and

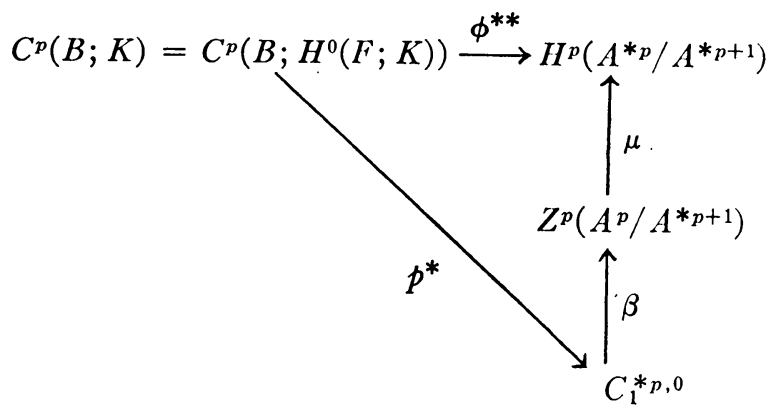

(4) Indices, attached to the homomorphisms $\lambda, \mu$ of $\mathrm{S} 1$, and to $\beta$ of $\mathrm{S} 2$, indicating the groups on which they operate have not been included, since these groups will be clear from the context in which $\lambda, \mu$ and $\beta$ occur subsequently.

(5) Cf. the cohomology analogues, briefly indicated by Serre, of the arguments of $[9, \S \S 2,7]$, in respect of the groups $R^{*}=A^{*} / A^{* 1}$, and $S_{p}^{*}=E^{* p, 0}$. 
where $C_{1}^{*_{p, 0}}$ is the subgroup formed of homogeneous elements of degree $p$ in $C_{1}^{*}$.

S3. Thirdly, the assumption that $\pi_{1}(B)$ operates trivially on $H^{*}(F, K)$ implies that $C^{0}\left(B ; H^{*}(F ; K)\right)=Z^{0}\left(B ; H^{*}(F ; K)\right)=I^{*}(F ; K)$. Thus for all $\mathrm{x} \in H^{*}(F ; K), \phi^{* *} \mathrm{x}$ is a $d_{1}^{*}$-cocycle in $E_{1}^{*_{0}}=I I^{*}\left(A^{*} / A^{* 1}\right)$, i.e., $d_{1}^{*} \phi^{* *} \mathrm{x}=0$. In consequence, given $\mathrm{x} \in H^{*}(F ; K)$ and hence $\phi^{*{ }_{x}} \in I^{*}\left(A^{*} / A^{* 1}\right)$, the element $y \in C_{1}^{* 0}$ such that $\mu \beta y=\phi^{* *}{ }_{x}$ can in fact be chosen so that $d y \in A^{* 2} \subset A^{* 1}$, where $d$ is the coboundary operator in $E$.

S4. Finally, the choice of a ring for coefficients enables one to define multiplicative structures in $E_{1}^{*}$ and $C^{*}\left(B ; H^{*}(F ; K)\right)$ such that $\phi^{* *}$ and $\psi^{* *}$ are multiplicative isomorphisms. The further assumption that $H^{q}(F ; K)$ is free and of finite type for all $q>0$ implies that $C^{*}\left(B ; H^{*}(F ; K)\right)$ can be identified with $C^{*}(B ; K) \otimes_{K} H^{*}(F ; K)$; in which case the product of $b \otimes x \in C^{p}(B ; K) \otimes_{K} H^{q}(F ; K)$ and $b^{\prime} \otimes x^{\prime} \in C^{p^{\prime}}(B ; K) \otimes H^{q^{\prime}}(F ; K)$ is of course given by

$$
(b \otimes x)\left(b^{\prime} \otimes x^{\prime}\right)=(-1)^{q p^{\prime}}\left(b b^{\prime} \otimes \mathbf{x x}^{\prime}\right),
$$

where $b b^{\prime}$ and $\mathbf{x x}^{\prime}$ are the usual cup products of $b$ and $b^{\prime}$, and $\mathbf{x}$ and $\mathbf{x}^{\prime}$, respectively, in $C^{*}(B ; K)$ and $H^{*}(F ; K)$, respectively.

It should be noted that if $x \in C_{1}^{*} p, q$ and $y \in C_{1}^{* p^{\prime}, q^{\prime}}$, then $\mu \beta\left(x \cup_{y}\right)$ $=\mu \beta(x) \cup_{\mu \beta}(y)$, where $\mu, \beta$ are as defined in S1, S2 above $\left({ }^{4}\right)$, and $x \cup_{y}$ $\in C_{1}^{* p+p^{\prime}, q+q^{\prime}}$, denotes the cup product, in $C^{*}(E ; K)$, of $x$ and $y$.

Consider now the method of Hirsch [7] in this context. As above, identify $C^{p}\left(B ; H^{*}(F ; K)\right)$ with $C^{p}(B ; K) \otimes_{K} H^{*}(F ; K)$, and in addition identify $C^{0}(B ; K) \otimes_{K} H^{*}(F ; K)$ with $H^{*}(F ; K)$. Since $I^{*}(F ; K)$ is assumed free, one can define a homomorphism

$$
u: H^{*}(F, K) \rightarrow C_{1}^{* 0} \rightarrow C^{*}(E ; K),
$$

which preserves degrees and makes commutative the diagram

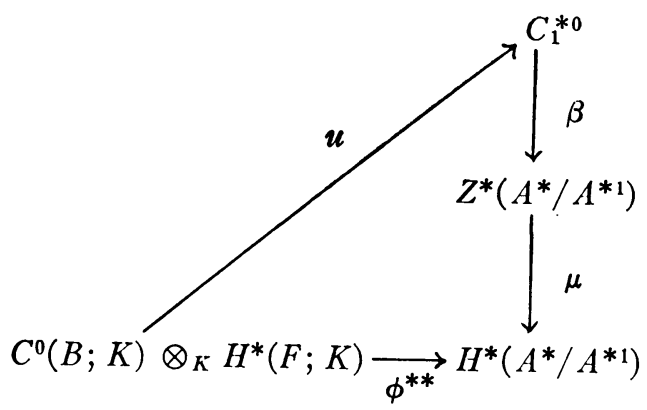

Moreover, as noted in S3 above, $u$ may be so chosen that $d u(\mathbf{x}) \in A^{* 2}$, where $d$ denotes the coboundary operator in $E$.

Recall from $\mathrm{S} 1$ and $\mathrm{S} 2$ that $i^{*}: C^{*}(E ; K) \rightarrow C^{*}(F ; K)=C^{0}\left(B ; C^{*}(F ; K)\right)$ is 
induced by the injection $i: F \rightarrow E$, and that $\lambda: C^{0}\left(B ; Z^{*}(F ; K)\right)$ $\rightarrow C^{0}\left(B ; H^{*}(F ; K)\right.$ ), (or equivalently $\lambda: Z^{*}(F, K) \rightarrow H^{*}(F, K)$ ), is the natural homomorphism mapping cocycles to cohomology classes. In these terms we have the following lemma concerning $u$.

Lemma 1. The homomorphism $u$ is a monomorphism with left inverse $\lambda i^{*}$.

Certainly $u$ is a monomorphism, for $\phi^{* *}$ has an inverse $\psi^{* *}$, so that for all $\mathbf{x} \in H^{*}(F ; K), \psi^{* *} \mu \beta u(\mathbf{x})=\mathbf{x}$. But as was seen above, in S2,

$$
\psi^{*} \beta=i^{*}: C_{1}^{* 0} \rightarrow Z^{*}(F ; K),
$$

so that

$$
\begin{aligned}
\mathbf{x} & =\psi^{* *} \mu \beta u(\mathrm{x}) \\
& =\lambda \psi^{*} \beta u(\mathbf{x})=\lambda i^{*} u(\mathrm{x}),
\end{aligned}
$$

as required.

This then is the first step in the Hirsch method, namely to define a monomorphism $u: H^{*}(F ; K) \rightarrow C^{*}(E ; K)$ such that $d u(\mathbf{x}) \in A^{* 2}$ for all $\mathbf{x} \in H^{*}(F ; K)$, and such that the composite homomorphism

$$
H^{*}(F ; K) \stackrel{u}{\rightarrow} C^{*}(E ; K) \stackrel{i^{*}}{\rightarrow} C^{*}(F ; K)
$$

maps a cohomology class $\mathrm{x}$ into a representative cocycle of $\mathbf{x}$.

The second step in the method is to extend $u: H^{*}(F ; K) \rightarrow C^{*}(E ; K)$ to a homomorphism

$$
u: C^{p}(B ; K) \otimes_{K} H^{*}(F ; K) \rightarrow C^{*}(E ; K),
$$

for all $p \geqq 0$. This is accomplished by setting

$$
u(b \otimes \mathbf{x})=p^{*} b \cup u(x),
$$

for all $b \in C^{*}(B ; K)$ and all $\mathrm{x} \in H^{*}(F ; K)$, where as usual $p^{*}: C^{*}(B ; K)$ $\rightarrow C^{*}(E ; K)$ is induced by the projection $p: E \rightarrow B$, and $\cup$ denotes the cup product in $C^{*}(E ; K)$. Notice $u$ preserves the (total) degrees.

Again with the same notation as in $\mathrm{S} 2$, namely $\beta: C_{1}^{*}{ }_{p} \rightarrow Z^{*}\left(A^{*_{p}} / A^{*_{p+1}}\right)$, $\mu: Z^{*}\left(A^{* p} / A^{* p+1}\right) \rightarrow H^{*}\left(A^{*_{p}} / A^{*_{p+1}}\right)$, we have the following lemma concerning $u$, and demonstrating the properties of $u$ which are carried over to $u$ by the extension.

LEMMA 2. The homomorphism $u$ is such that

$$
\boldsymbol{u}\left(C^{p}(B ; K) \otimes_{K} H^{*}(F ; K)\right) \subset C_{1}^{* p}
$$

and morcover the following diagram is commutative: 


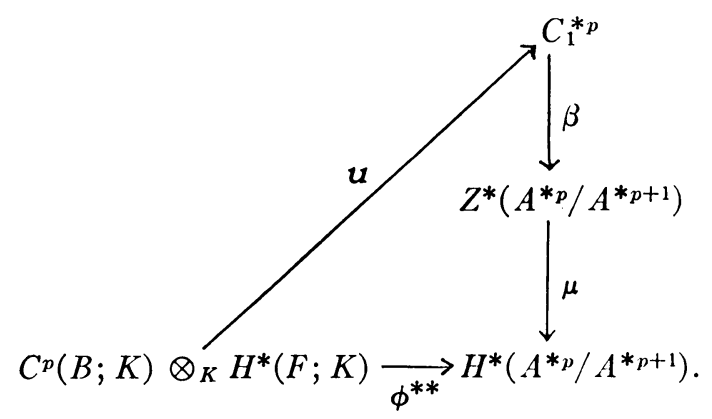

Of course it follows immediately from the second part of this lemma, since $\phi^{* *}$ has inverse $\psi^{* *}$, that $u$, like $u$, is a monomorphism with left inverse $\psi^{* *} \mu \beta$.

The first part of the lemma is an immediate consequence of the definition of $u$, for if $b \in C^{p}(B ; K), \mathrm{x} \in H^{*}(F ; K)$, as we have seen above, in $\mathrm{S} 2, p^{*} b \in C_{1}^{* p}$, and $u(x) \in C_{1}^{*}$. Thus $p^{*} b \cup u(x) \in C_{1}^{*}$. The second part follows equally simply from the facts previously noted. Thus, recalling the multiplicative properties of $\mu \beta$, given in S4,

$$
\begin{aligned}
\phi^{* *}(b \otimes \mathbf{x}) & =\phi^{* *}((b \otimes 1)(1 \otimes \mathbf{x})) \\
& =\phi^{* *}(b \otimes 1) \cup \phi^{* *}(1 \otimes \mathbf{x}) \\
& =\left(\mu \beta p^{*} b\right) \cup(\mu \beta u(x)) \\
& =\mu \beta\left(p^{*} b \cup u(\mathbf{x})\right) \\
& =\mu \beta u(b \otimes x) .
\end{aligned}
$$

We can now state and verify the assertion of the Hirsch method in the precise form suggested by $\mathrm{H}$. Cartan $\left(^{6}\right)$.

(i) The monomorphism $u$ can be chosen, as above, satisfying $\mu \beta u=\phi^{* *}$ (or equivalently $\left.\lambda i^{*} u=1\right)$ so that for all $\mathbf{x} \in H^{q}(F, K)$

$$
d u(x) \in u\left\{\sum_{2 \leqq r \leq q+1} C^{r}(B ; K) \otimes_{K} H^{q+1-r}(F ; K)\right\} .
$$

(ii) Moreover with this choice of $u$, the image

$$
u\left\{C^{*}(B ; K) \otimes_{K} H^{*}(F ; K)\right\} \subset C^{*}(E ; K)
$$

(6) See the review of [7] by H. Cartan (Math. Rev. vol. 16 (1955) p. 1142). There is an error in the statement of the properties of $\partial$ as given in the Reviewers Note, there. It is only $H^{q}(F ; K)$ identified with $C^{0}(B ; K) \otimes H^{q}(F ; K)$ which is mapped by $\partial$ into $C^{*}(B ; K)$ $\otimes \sum_{m<q} H^{m}(F ; K)$. The present reconstruction of the proof of the Hirsch result was produced in an attempt to prove the assertion of Professor Cartan, and I am grateful to him for sending me a manuscript correcting his review and reconstructing the Hirsch method in the context of C.S.S. complexes and fibrations in the sense of Kan. The proof given here seems to agree in general outline with that of Professor Cartan. 
is a graded sub-K-module of $C^{*}(E ; K)$ which is stable under the coboundary operator $d$ in $C^{*}(E ; K)$.

(iii) In consequence, carrying back to $C^{*}(B ; K) \otimes_{K} H^{*}(F ; K)$ the coboundary operator $d$ of $C^{*}(E ; K)$, there is obtained the graded cohomology $K$-module of $C^{*}(B ; K) \otimes_{K} H^{*}(F ; K)$ which is isomorphic with the graded cohomology $K$ module $H^{*}(E ; K)$.

The fact that part (i) of the assertion implies part (ii), and hence part (iii), is straightforward; indeed more will be seen to be true. For let $b \otimes x \in C^{p}(B ; K) \otimes_{K} H^{q}(F ; K)$ where, as is implied by part (i) of the assertion,

$$
d u(\mathrm{x})=\sum_{2 \leqq r \leq q+1} \sum_{i=1}^{n_{r}} p^{*} b_{r}^{i} \cup u\left(\mathbf{x}_{r}^{i}\right)
$$

for some $b_{r}^{i} \otimes \mathbf{x}_{r}^{i} \in C^{r}(B ; K) \otimes H^{q+1-r}(F ; K)$. Then

$$
\begin{aligned}
d u(b \otimes x) & =d\left(p^{*} b \cup u(\mathrm{x})\right) \\
& =d p^{*} b \cup u(\mathrm{x})+(-1)^{p} p^{*} b \cup d u(\mathrm{x}) \\
& =p^{*} \partial_{B} b \cup u(\mathrm{x})+(-1)^{p} \sum_{2 \leqq r \leq q+1} \sum_{i=1}^{n_{r}} p^{*} b \cup p^{*} b_{r}^{i} \cup u\left(\mathbf{x}_{r}^{i}\right) \\
& =p^{*} \partial_{B} b \cup u(\mathrm{x})+(-1)^{p} \sum_{2 \leqq r \leq q+1} \sum_{i=1}^{n_{r}} p^{*}\left(b b_{r}^{i}\right) \cup u\left(\mathbf{x}_{r}^{i}\right) \\
& =u\left\{\partial_{B} b \otimes \mathbf{x}+(-1)^{p} \sum_{2 \leqq r \leq q+1} \sum_{i=1}^{n_{r}} b b_{r}^{i} \otimes x_{r}^{i}\right\} .
\end{aligned}
$$

Thus in case $b \otimes \mathbf{x} \in C^{p}(B ; K) \otimes H^{q}(F ; K)$,

$$
d u(b \otimes x) \in u\left\{\sum_{p \leq r \leq p+q} C^{r+1}(B ; K) \otimes_{K} H^{p+q-r}(F ; K)\right\},
$$

so that the stability under $d$, not only of $u\left\{C^{*}(B ; K) \otimes_{K} H^{*}(F ; K)\right\}$, which proves part (ii) of the assertion, but also of

$$
u\left\{\sum_{r \geq i ; s \leq j} C^{r}(B ; K) \otimes_{K} H^{s}(F ; K)\right\},
$$

follows. Therefore, using Lemma 2, the coboundary operator $d$ of $C^{*}(E, K)$ can be carried back to $C^{*}(B ; K) \otimes_{K} H^{*}(F ; K)$ and, in particular, $\boldsymbol{u}$ becomes a cochain mapping of $C^{*}(B ; K) \otimes_{K} H^{*}(F ; K)$ into $C^{*}(E ; K)$. Hence by a familiar argument $\left({ }^{7}\right)$, using the filtration

$$
F_{i}\left\{C^{*}(B ; K) \otimes_{K} \sum_{s \leq j} H^{s}(F ; K)\right\}=\sum_{r \geq i ; s \leq j} C^{r}(B ; K) \otimes_{K} H^{s}(F ; K),
$$

$\left({ }^{7}\right)$ Cf., e.g. the Hint of Example 7, p. $74\lceil 3\rceil$. 
(which is preserved by $\boldsymbol{u}$ ), the five lemma, and a descending induction on $i$, one proves that $u$ not only induces an isomorphism between $H^{*}\left(C^{*}(B ; K)\right.$ $\left.\otimes_{K} H^{*}(F ; K)\right)$ and $H^{*}(E ; K)$, as required for part (iii) of the assertion, but also between $H^{r}\left(F_{i}\left\{C^{*}(B ; K) \otimes_{K} \sum_{s \leq j} H^{s}(F ; K)\right\}\right)$ and $H^{r}\left(A^{* i}\right)$ for all $r \leqq i+j$. Moreover, of course, to prove this last fact, $u$ need only be assumed to satisfy part (i) of the assertion on $\sum_{q_{\Sigma} j} H^{q}(F, K)$.

Finally, to the proof of part (i) of the assertion! It will be by induction on the dimension $s$ of $H^{s}(F ; K)$. Certainly, in case $s=0, u$ can be defined on $H^{0}(F ; K)$ so as to satisfy the assertion. Suppose therefore that $u: H^{s}(F ; K)$ $\rightarrow C^{s}(E ; K)$ has been chosen to satisfy part (i) of the assertion for all $s \leqq j$. We require to define $u$ on each basis element of $H^{j+1}(F ; K)$. Let $\mathbf{x}$ be such a basis element, and let $u(x)$ be chosen (as it may be, by S3 above) so that $d u(\mathbf{x}) \in A^{* 2}$, and such that, as in Lemma $1, \lambda i^{*} u(\mathbf{x})=\mathbf{x}$. Consider $d u(\mathbf{x})$. It is a cocycle of dimension $j+2$, in $A^{* 2}$. Thus it defines a cohomology class in $H^{i+2}\left(A^{* 2}\right)$. But, as we have seen, since $u$ satisfies the first part of the Hirsch assertion on $\sum_{s \leq j} H^{s}(F ; K), \boldsymbol{u}$ induces an isomorphism

$$
H^{i+2}\left(\sum_{r \geq 2} C^{r}(B ; K) \otimes_{K} H^{i+2-r}(F ; K)\right) \approx H^{i+2}\left(A^{* 2}\right) .
$$

Thus

$$
d u(x)=u(y)+d z,
$$

for some $y \in \sum_{r \geq 2} C^{r}(B ; K) \otimes_{K} H^{i+2-r}(F ; K)$, and $z \in A^{* 2}$ of degree $q+1$. If we now alter $u$ on $H^{j+1}(F ; K)$ to $u^{\prime}$, say, where $u^{\prime}(\mathbf{x})=u(\mathrm{x})-z$, then $u^{\prime}$ not only satisfies the same conditions as $u$ in that $d u^{\prime}(\mathbf{x}) \in A^{* 2}$ and $\lambda i^{*} u^{\prime}(\mathbf{x})=\mathbf{x}$, but also fully satisfies part (i) of the Hirsch assertion. Thus taking $u^{\prime}$ in place of $u$ on $H^{q+1}(F ; K)$, the assertion is proved.

Note 2.1. Professor H. Cartan has pointed out $\left(^{8}\right)$ that the Hirsch result holds even if the basis of $H^{q}(F ; K)$ is not finite for all $q \geqq 0$, on condition that one replaces $C^{*}(B ; K) \otimes_{K} H^{*}(F ; K)$ by Hom $\left(C_{*}(B), H^{*}(F ; K)\right)$, where $C_{*}(B)$ denotes the singular chains of the base. The arguments used above can be adapted to prove the result under these more general conditions. In two places the argument needs some expansion. Firstly in the extension of $u$ to $\boldsymbol{u}$, the straightforward cup product definition $u(b \otimes \mathbf{x})=p^{*} b \cup u(x)$ has to be replaced by a homomorphism $u$ : Hom $\left(C_{*}(B), H^{*}(F ; K)\right) \rightarrow C^{*}(E ; K)$ defined by composing the homomorphisms $\left({ }^{9}\right) \quad u^{\prime}: \operatorname{Hom}\left(C_{*}(B), H^{*}(F ; K)\right)$

(8) Math. Rev. vol. 16 (1955) p. 1142.

${ }^{(9)}$ In order that $u$ so defined shall induce precisely the homomorphism $\phi^{*}$ of Serre, as is required, it appears necessary also to include a "twisting" homomorphism $C_{*}(E) \otimes C_{*}(E)$ $\rightarrow C_{*}(E) \otimes C_{*}(E)$, given by $e_{1} \otimes e_{2} \rightarrow(-1)^{p q} e_{2} \otimes e_{1}$. This is avoided above in choosing the factorization $(b \otimes \mathbf{x})=(b \otimes \mathbf{1})(1 \otimes \mathbf{x})$, in $C^{*}(B ; K) \otimes H^{*}(F ; K)$, rather than $(b \otimes \mathbf{x})= \pm(1 \otimes \mathbf{x})(b \otimes \mathbf{1})$. This last factorization is effectively forced upon one in the general case if Serre's $\phi^{*}$ is required. 
$\rightarrow \operatorname{Hom}\left(C_{*}(B), C^{*}(E ; K)\right)$, (induced by $\left.u\right), p^{*}: \operatorname{Hom}\left(C_{*}(B), C^{*}(E ; K)\right)$ $\rightarrow$ Hom $\left(C_{*}(E), C^{*}(E ; K)\right.$ ), (induced by $p: E \rightarrow B$ ), and the usual (cohomology) transpose of the chain mapping $C_{*}(E) \rightarrow C_{*}(E) \times C_{*}(E) \rightarrow C_{*}(E) \otimes C_{*}(E)$ which defines the cup-products. Secondly, this more complicated definition of $u$ requires that slightly more care is taken in proving that part (i) of the Hirsch assertion implies parts (ii) and (iii). The argument given above is replaced by one involving the new definition of $u$ in terms of the homomorphisms which compose it, and a commutative diagram, involving the groups and homomorphisms involved, seems the standard, and simplest, way of coping with the extra labor involved in proving (i) implies (ii).

Professor Cartan has also pointed out to me privately $\left({ }^{6}\right)$, that the Hirsch result is equally well proved in the category of C.S.S. complexes for fibrations of such complexes in the sense of Kan (cf. J. C. Moore, Mimeographed Lecture Notes, Princeton, 1956). Here it seems convenient, right from the start, to define the homomorphism analogous to the Serre homomorphism of the $E_{0}$ term of the homology spectral sequence, i.e. $\phi=E_{0} \rightarrow C_{*}(B) \otimes C_{*}(F)$, in terms of the diagonal mapping, $E \rightarrow E \times E$, and the projection, $E \rightarrow B$, and injection of the fibration. Thus in this case the fact that when $u$ is extended to $u$ it induces the homomorphism analogous to $\phi^{*}$ : Hom $\left(C_{*}(B), H^{*}(F ; K)\right)$ $\rightarrow E_{1}^{*}$, in the cohomology spectral sequences, is immediate.

Note 2.2. Professor E. L. Brown has also pointed out to me that the homology analogue of the Hirsch result is also true, i.e. that, assuming $H_{*}(F ; K)$ to have a homogeneous $K$-basis, one can find a homomorphism $\mu: C_{*}(E ; K) \rightarrow H_{*}(F ; K)$ which can be extended to a homomorphism $\boldsymbol{u}: C_{*}(E ; K)$ $\rightarrow C_{*}(B ; K) \otimes_{K} H_{*}(F ; K)$ inducing a graded isomorphism $H_{*}(E, K)$ $\approx H_{*}\left(C_{*}(B ; K) \otimes_{K} H_{*}(F ; K)\right.$ ), (and hence an isomorphism in cohomology,

$$
\left.H^{*}\left(C_{*}(B ; K) \otimes_{K} H_{*}(F ; K)\right)=H^{*}\left(C^{*}\left(B ; H^{*}(F ; K)\right)\right) \approx H^{*}(E ; K)\right) .
$$

This also appears to be true in the category of C.S.S. complexes, for Kan fibrations.

3. The Hirsch coboundary operator and cohomological operations. Proof of the main result. As stated in the introduction, the aim of this section is to interpret the Hirsch coboundary formula, given in the last section, in terms of the theory of cohomological operations, in case the fibre $F$ of the fibre space $(E, B, F)$ is of type $\Re(\pi, n)$.

I require first to recall the notion of transgression and of transgressive elements in a fibre space. There are of course several equivalent definitions of these notions in a fibre space $(E, B, F)$ in the sense of Serre. One of the most useful states that $\mathrm{x} \in H^{s-1}(F ; K)$ is transgressive if there exists a cochain $c \in C^{s-1}(E ; K)$, (the transgression cochain), such that $i^{*} c$ is a representative cocycle of $\mathbf{x}$ and such that $d c=p^{*} z$ for some $z \in C^{s}(B ; K)$, where as usual $i^{*}: C^{s-1}(E ; K) \rightarrow C^{s-1}(F ; K)$ and $p^{*}: C^{s}(B ; K) \rightarrow C^{s}(E ; K)$ are induced respectively by the injection and projection mappings $i: F \rightarrow E, p: E \rightarrow B$ associated 
with $E$. Alternatively the transgression may be defined as the differential $d_{s}: E_{s}^{*_{0, s-1}} \rightarrow E_{s}^{*_{s, 0}}$ of the cohomology spectral sequence associated with $E$; or by means of the homomorphisms

$$
H^{s-1}(F ; K) \stackrel{\delta}{\rightarrow} H^{s}(E ; F ; K) \stackrel{p^{\natural}}{\leftarrow} H^{s}(B ; K),
$$

where $\delta$ here denotes the usual coboundary operator associated with the pair $(E, F)$, and $p^{\natural}$ is induced by $p: E \rightarrow B$. In the former case an element $x \in H^{s-1}(F ; K)=E_{1}^{*_{0,8-1}}$ is transgressive if it is a cocycle for all the differentials $d_{1}, d_{2}, \cdots, d_{s-1}$; in the latter case the transgression maps a subgroup of $H^{s-1}(F ; K)$ homomorphically onto a factor group of $H^{s}(B ; K)$, i.e. for $\mathbf{x} \in H^{s-1}(F ; K)$ to be transgressive, there must exist $z \in H^{s}(B ; K)$ such that $\delta \mathbf{x}=p^{\natural} z$, where $\boldsymbol{z}$ is of course called the transgression of $\mathbf{x}$.

Since $H^{s}(\pi, n ; K)=0$ for $0<s<n$, it follows that when the fibre $F$ in the fibre space $(E, B, F)$ is of type $\Re(\pi, n)$ any element of $H^{n}(F ; K)$ is transgressive, and the transgression homomorphism $\tau^{*}$ maps $H^{n}(F ; K)$ into $H^{n+1}(B ; K)$. In particular the fundamental cohomology class, $b^{n}$, of $F$ (i.e. the element of $H^{n}(\pi, n ; \pi)$ which corresponds to the identity automorphism under the isomorphism $\left.H^{n}(\pi, n ; \pi) \approx \operatorname{Hom}(\pi, \pi)\right)$ is transgressive. The characteristic cohomology class $k^{n+1} \in H^{n+1}(B ; \pi)$ of the fibre space is defined by $\boldsymbol{k}^{n+1}$ $=-\tau^{*} b^{n}$.

In the reverse direction to the transgression, i.e. mapping homomorphically a subgroup of $H^{s}(B ; K)$ onto a factor group of $H^{s-1}(F ; K)$ by means of the homomorphisms

$$
H^{s-1}(F ; K) \stackrel{\delta}{\rightarrow} H^{s}(E, F ; K) \stackrel{p^{\natural}}{\leftarrow} H^{s}(B ; K),
$$

we have the cohomology suspension $\sigma^{*}$.

If $E$ is contractible, $\delta$ is an isomorphism and $\sigma^{*}=\delta^{-1} p^{\natural}: H^{s}(B ; K)$ $\rightarrow I^{s-1}(F ; K)$, so that under this circumstance an element $\mathrm{x} \in H^{s-1}(F ; K)$ is transgressive if and only if $x \in \sigma^{*} H^{s}(B ; K)$. This will be true of course in the case when $E$ is the space of paths in $B$ with fixed initial point. If in this case the fibre space is further specialized by taking $B$ to be a space of type $\Re(\pi, n+1)$, so that $F$ (being the space of loops on $B$ ) is of type $\mathscr{K}(\pi, n)$, then as is well known, the suspension $\sigma^{*}$ is an isomorphism in dimensions $<2 n$, i.e.

$$
\sigma^{*}: H^{s+1}(\pi, n+1 ; K) \approx H^{s}(\pi, n ; K) \quad 0<s<2 n .
$$

Thus in this case all the elements of $H^{s}(\pi, n ; K)$ for $s<2 n$ are transgressive; indeed in these dimensions the transgression homomorphism $\tau^{*}: H^{s}(\pi, n ; K)$ $\rightarrow H^{s+1}(\pi, n+1 ; K)$ is the inverse of the suspension homomorphism $\sigma^{*}$ : $H^{s+1}(\pi, n+1 ; K) \rightarrow H^{s}(\pi, n ; K)$.

In order to make use of these facts in the case which is of interest here, i.e. when $B$ is arbitrary and $F$ is of the type $\mathfrak{K}(\pi, n)$, we require the notions 
of cohomological operations. I next recall the formulation of this theory as given by H. Cartan [2]. Notice that the formulation given by Cartan in [2] is based on a simplicial and not cubical foundation. Inasmuch as I have used cubical cohomology theory in $(E, B, F)$, in what follows I make implicit use of the Eilenberg-MacLane theorem $\left({ }^{10}\right)$ on the equivalence of the singular simplicial and singular cubical cohomology theories.

The cohomological operations, then, are in a one-one correspondence with the elements of $H^{q}(\pi, n ; K), q \geqq 0$. Given $\mathrm{x} \in H^{q}(\pi, n ; K)$ and any topological space $X$, there is an operation corresponding to $x$ which is a linear mapping

$$
T(\mathbf{x}): H^{n}(X ; \pi) \rightarrow H^{q}(X ; K) .
$$

If $b^{n}$ is the fundamental cohomology class in $H^{n}(\pi, n ; \pi)$ then taking $X$ to be a space of type $\mathscr{K}(\pi, n), T(x)$ defines a linear mapping

$$
T(\mathrm{x}): H^{n}(\pi, n ; \pi) \rightarrow H^{q}(\pi, n ; K)
$$

such that $\mathbf{x}=T(\mathbf{x}) \boldsymbol{b}^{n}$.

The operations $T(x)$ are also defined in relative cohomology. Thus if $Y$ is a subspace of $X$, there is a linear mapping

$$
T(\mathrm{x}): H^{n}(X, Y ; \pi) \rightarrow H^{q}(X, Y ; K)
$$

associated with each $\mathrm{x} \in H^{q}(\pi, n ; K)$.

That the theory of cohomological operations enables one to make use of the ideas of transgression noted above, and indeed to determine transgressive elements in a fibre space with fibre of type $\mathscr{K}(\pi, n)$, is clear in the light of the proof of the following lemma.

Lemua. If $\mathrm{x} \in H^{q}(\pi, n ; K)$ is transgressive in the fibre space of paths on a space of type $\nVdash(\pi, n+1)$, then it is also transgressive in any fibre space with fibre of type $\mathfrak{K}(\pi, n)$.

The lemma adds a further point to the likeness of the fibre space of paths on a space of type $\mathscr{K}(\pi, n+1)$ to a universal classifying space for fibre spaces with fibre of type $\mathscr{K}(\pi, n)$. The elements of $H^{q}(\pi, n ; K)$ which are transgressive in the space of paths may be said to be "universally transgressive."

The proof of the lemma is straightforward. If $x \in H^{q}(\pi, n ; K)$ is transgressive in the space of paths on a space of type $\mathscr{K}(\pi, n+1)$, then $\mathrm{x}=\sigma^{*} \boldsymbol{y}$ for some $y \in H^{q+1}(\pi, n+1 ; K)$. Associated with $\mathrm{x}$ and $\mathrm{y}$ are cohomological operations $T(\mathbf{x})$ and $T(\boldsymbol{y})$ respectively. It follows immediately from the results of $\mathrm{H}$. Cartan( $\left.{ }^{11}\right)$, that for any fibre space $(E, B, F)$, there is a commutative diagram
(10) See [6].
(11) See [2]. 


$$
\begin{aligned}
& H^{n}(F ; \pi) \stackrel{\delta}{\rightarrow} H^{n+1}(E, F ; \pi) \stackrel{p^{\natural}}{\leftarrow} H^{n+1}(B ; \pi) \\
& T(\mathrm{x}) \downarrow \\
& H^{q}(F ; K) \underset{\delta}{\rightarrow} H^{q+1}(E, F ; K) \underset{p^{\natural}}{\leftarrow} H^{q+1}(B ; K) .
\end{aligned}
$$

Now if $F$ is of type $\mathcal{K}(\pi, n), \mathbf{x} \in H^{q}(F ; K)=H^{q}(\pi, n ; K)$ is of the form $T(\mathbf{x}) \boldsymbol{b}^{n}$. Moreover $-\delta \boldsymbol{b}^{n}=\boldsymbol{p}^{\natural} \boldsymbol{k}^{n+1}$, where $\boldsymbol{k}^{n+1}$ is the characteristic class of $E$. Thus

$$
\delta \mathrm{x}=\delta T(\mathrm{x}) b^{n}=T(\mathrm{y}) \delta b^{n}=-T(y) p^{\natural} k^{n+1}=p^{\natural}\left(-T(y) k^{n+1}\right),
$$

i.e. $\mathbf{x}$ is transgressive in $(E, B, F)$.

We are now in a position to deduce from the Hirsch assertion of the last section the main result, which for convenience I restate.

TheOREM. In case $0<m<2 n$, the coboundary operator

$$
\partial: C^{p}(B ; K) \otimes_{K} H^{q}(\pi, n ; K) \rightarrow \sum_{p \leq r \leq p+q} C^{r+1}(B ; K) \otimes_{K} H^{p+q-r}(\pi, n ; K),
$$

of the Theorem of Hirsch, can be defined by setting

$$
\begin{aligned}
& \partial(b \otimes 1)=\partial_{B} b \otimes 1, \\
& \partial(b \otimes x)=\partial_{B} b \otimes x-(-1)^{p}\left(b \cup T(y) k^{n+1}\right) \otimes 1,
\end{aligned}
$$

for all $b \in C^{p}(B ; K)$, and all basis elements $\mathbf{x} \in I I^{q}(\pi, n ; K)$, where $p+q=m$, $\sigma^{*} \boldsymbol{y}=\mathbf{x}, 1$ denotes the unit element in $H^{*}(\pi, n, K)$, and $T(\boldsymbol{y}) k^{n+1}$ denotes $a$ representative cocycle of $T(y) k^{n+1}$.

By definition

$$
\boldsymbol{u}: C^{*}(B ; K) \otimes_{K} H^{0}(F ; K) \rightarrow C^{*}(E ; K)
$$

coincides with

$$
p^{*}: C^{*}(B ; K) \rightarrow C^{*}(E ; K) .
$$

Suppose therefore that a basis element $\mathrm{x} \in H^{q}(F ; K)$ is transgressive, with transgression $z \in H^{q+1}(B ; K)$. Given any cocycle $z$ in the cohomology class $z$, there may be chosen a cochain $c$ in $C^{s}(E ; K)$ such that $d c=p^{*} z$ and such that $i^{*} c$ is a representative cocycle of $x$. In this case therefore, in the Hirsch method we may choose $u(x)=c$, whence $d u(x)=u(z)$, and the requirements of the method are easily seen to be satisfied. Moreover, carrying back the coboundary operator $d: C^{*}(E ; K) \rightarrow C^{*}(E ; K)$ to $C^{*}(B ; K) \otimes_{K} H^{*}(F ; K)$, evidently ( $\left.{ }^{12}\right)$

$\left.{ }^{(12}\right)$ In terms of the transgression, the coboundary operator $\partial$ is, then, the same on the basis elements $\mathrm{x}$ as that of A. Borel [1, Chapter VI, \$24]. However because of the nonanticommutativity of $C^{*}(E ; K)$, even if $H^{*}(\pi, n ; K)$ is an anticommutative algebra with transgressive generators, it is not sufficient to define $\partial$ on these generators alone, if $\partial$ is required in dimensions $>2 n-1$. One cannot in fact, in general, choose $u(x y)=u(x) u(y)$ if $\mathbf{x}$ and $y$ are generators of $H^{*}(\pi, n ; K)$. 


$$
\partial(1 \otimes x)=z \otimes 1 .
$$

Now in case the fibre $F$ of $(E, B, F)$ is of type $\Re(\pi, n)$ we have seen that all the basis elements of $H^{*}(\pi, n ; K)$ are transgressive in $(E, B, F)$ up to dimension $2 n-1$, the transgression of $\mathrm{x} \in H^{q}(\pi, n ; K)$ being $-T(y) k^{n+1}$, where $\sigma^{*} y=x$. So in this case we may choose for $z$ any cocycle $-T(y) k^{n+1}$ in the class $-T(y) k^{n+1}$. This concludes the proof of the more difficult part of the theorem. The fact that

$$
\partial(b \otimes 1)=d_{B} b \otimes 1,
$$

for all $b=C^{*}(B ; K)$ is of course trivial and follows immediately from the fact noted above that $u$ coincides with $p^{*}$ on $b \otimes 1$.

The reader may note moreover that in calculating the groups $H^{m}\left(C^{*}(B ; K) \otimes_{K} H^{*}(\pi, n ; K)\right)$, for $m \leqq 2 n-1$, only the coboundary operator in $H^{q}(\pi, n ; K)$ for dimensions $q \leqq 2 n-1$, is required. For denoting by $\partial$, as usual, the Hirsch coboundary operator in $C^{*}(B ; K) \otimes_{K} H^{*}(\pi, n ; K)$, we have

$$
\partial\left\{C^{p}(B ; K) \otimes_{K} H^{q}(\pi, n ; K)\right\} \subset \sum_{p \leqq r \leqq p+q} C^{r+1}(B ; K) \otimes_{K} H^{p+q-r}(\pi, n ; K)
$$

so that no coboundaries under $\partial$ arise in dimensions $\leqq m$ from terms of $C^{p}(B ; K) \otimes_{K} H^{q}(\pi, n ; K)$ with $p+q>m$. Thus the knowledge of $\partial$ given by the main result certainly enables one to compute the cohomology groups of $E$ in dimensions $0 \leqq m<2 n$.

Note 3.1. In view of the facts observed in Note 2.1 above, it is possible to extend the above arguments to include the case when $\pi$ is not finitely generated, so that $H^{*}(F ; K)=H^{*}(\pi, n ; K)$ need not be of finite type in each dimension. The assignment $\mathrm{x} \rightarrow-T(\boldsymbol{y}) k^{n+1}$, for all basis elements $\mathrm{x} \in H^{*}(\pi, n ; K)$, (where as usual $\mathbf{x}=\sigma^{*} y$ ), together with the cup product in $B$, yields a homomorphism

$$
\partial_{F}: \operatorname{Hom}\left(C_{p}(B), H^{q}(\pi, n ; K)\right) \rightarrow \operatorname{Hom}\left(C_{p+q+1}(B), H^{0}(\pi, n ; K)\right)
$$

for all $0<p+q<2 n$. The Hirsch coboundary operator $\partial$ in the dimension range $0<m<2 n$ is then determined by setting

$$
\begin{aligned}
\partial & =\partial_{B}+\partial_{F}: \sum_{p+q=m} \operatorname{Hom}\left(C_{p}(B), H^{q}(\pi, n ; K)\right) \\
& \rightarrow \sum_{p+q=m+1} \operatorname{Hom}\left(C_{p}(B), H^{q}(\pi, n ; K)\right) .
\end{aligned}
$$

4. Application of results. In this section results of [4] are reobtained by use of the main result. The method generalizes a group construction of Eilenberg-MacLane.

As before, let $(E, B, F)$ be a fibre space with fibre $F$ of type $\kappa(\pi, n)$ and characteristic class $k^{n+1} \in H^{n+1}(B ; \pi)$. The usual assumptions, enabling the Hirsch method to be applied, will be made. Thus $\pi_{1}(B)$ will operate trivially 
on the cohomology of the fibre $F$ and coefficients will be taken in a commutative ring $K$; the $K$-module $H^{*}(F ; K)=H^{*}(\pi, n ; K)$ will be assumed to have a homogeneous $K$-basis, finite in each dimension.

Consider the computation by means of the main result of $\S 3$, of the group $H^{n}\left(C^{*}(B ; K) \otimes_{K} H^{*}(\pi, n ; K)\right)$, which is of course isomorphic with the group $H^{n}(E ; K)$. The "cochain" group of $C^{*}(B ; K) \otimes_{K} H^{*}(\pi, n ; K)$, in dimension $n-1$, is (since $\left.H^{q}(\pi, n ; K)=0,0<q<n\right)$,

$$
C^{n-1}(B ; K) \otimes_{K} H^{0}(\pi, n ; K)=C^{n-1}(B ; K) .
$$

The Hirsch coboundary operator in this dimension reduces simply to the coboundary operator of $B$, i.e. $\partial_{B}: C^{n-1}(B ; K) \rightarrow C^{n}(B ; K)$. In dimension $n$, the cochain group of $C^{*}(B ; K) \otimes_{K} H^{*}(\pi, n ; K)$ is

$C^{n}(B ; K) \otimes_{K} H^{0}(\pi, n ; K)+C^{0}(B ; K) \otimes_{K} H^{n}(\pi, n ; K)=C^{n}(B ; K)+H^{n}(\pi, n ; K)$.

Since $H^{n}(\pi, n ; K)$ can be identified with $\operatorname{Hom}(\pi, K)$, this cochain group can be rewritten as

$$
C^{n}(B ; K)+\operatorname{Hom}(\pi, K) .
$$

To compute the Hirsch coboundary operator in this group we require the cohomological operations associated with $H^{n+1}(\pi, n+1 ; K)$. These are the homomorphisms

$$
\rho^{*}: H^{n+1}(B ; \pi) \rightarrow H^{n+1}(B ; K)
$$

induced by the homomorphisms $\rho \in \operatorname{Hom}(\pi, K)$. Indeed, after identifying $H^{n+1}(\pi, n+1 ; K)$ with $\operatorname{Hom}(\pi, K)$, (so that the suspension isomorphism $H^{n+1}(\pi, n+1 ; K) \approx H^{n}(\pi, n ; K)$ becomes the identity mapping), the operation associated with $\rho \in \operatorname{Hom}(\pi, K)=H^{n+1}(\pi, n+1 ; K)$ is precisely the homomorphism $\rho^{*}: H^{n+1}(B ; \pi) \rightarrow H^{n+1}(B ; K)$ induced by $\rho$. Thus if the cocycle $\rho k^{n+1}$ is chosen as a representative cocycle of the cohomology class $\rho^{*} k^{n+1}$, the Hirsch coboundary operator is defined in dimension $n$ by setting $\left({ }^{13}\right)$

$$
\partial(b, \rho)=\partial_{B} b-\rho k^{n+1} \in C^{n+1}(B ; K),
$$

for all pairs $(b, \rho)$ in the direct sum

$$
C^{n}(B ; K)+\operatorname{Hom}(\pi, K) .
$$

Thus the cocycles in dimension $n$, under $\partial$, are the pairs

$$
(b, \rho) \in C^{n}(B ; K)+\operatorname{Hom}(\pi, K),
$$

such that $\partial_{B} b=\rho k^{n+1}$, and the coboundaries are the elements of the group $\partial_{B} C^{n-1}(B ; K)$. The resulting cohomology group is thus precisely analogous to the group $E^{n}(k, G)$ constructed by Eilenberg-MacLane [8, Theorem II]. In

${ }^{(13)}$ It should be noted of course that $\rho$ need not be a basis element of $\operatorname{Hom}(\pi, K)$ in this definition. The choice of cocycle $\rho k^{n+1}$ in $\rho^{*} k^{n+1}$ for any $\rho$, is equivalent to choosing $\rho k^{n+1}$ in $\rho^{*} k^{n+1}$ for all basis elements $\rho$. 
fact, if $B$ is a space of type $K\left(\pi^{\prime}, m\right), n>m>1$, so that $E$ is a space with two nonva nishing homotopy groups, $\pi, \pi^{\prime}$, the group $H^{n}\left(C^{*}(B ; K) \otimes_{K} H^{*}(\pi, n ; K)\right)$ is exactly the Eilenberg-MacLane group $E^{n}\left(k^{n+1}, K\right)$ associated with $E$.

One readily verifies that $H^{n}(B, K)$ is a subgroup of

$$
H^{n}\left(C^{*}(B ; K) \otimes_{K} H^{*}(\pi, n ; K)\right)
$$

and that the correspondence $(b, \rho) \rightarrow \rho$ yields an epimorphism

$$
H^{n}\left(C^{*}(B ; K) \otimes_{K} H^{*}(\pi, n ; K)\right) \rightarrow \operatorname{Hom}(\pi, K),
$$

with kernel $H^{n}(B ; K)$. Thus we have the result of [4], that there exists an exact sequence

$$
0 \rightarrow H^{n}(B ; K) \rightarrow H^{n}(E ; K) \rightarrow \operatorname{Hom}(\pi, K) \rightarrow 0,
$$

which is of course to be expected in view of the analogy with the EilenbergMacLane result, [8, Theorem II].

The above construction has been given under the conditions usually imposed here. Evidently, in view of Note 3.1, it could perfectly well be carried through under the less stringent conditions of Note 2.1. For certainly

and

$$
\operatorname{Hom}\left(C_{n-1}(B), H^{0}(\pi, n ; K)\right)=C^{n-1}(B ; K),
$$

$\operatorname{Hom}\left(C_{0}(B), H^{n}(\pi, n ; K)\right)+\operatorname{Hom}\left(C_{n}(B), H^{0}(\pi, n ; K)\right)=\operatorname{Hom}(\pi, K)+C^{n}(B ; K)$

are the required cochain groups of $\operatorname{Hom}\left(C_{*}(B), H^{*}(\pi, n ; K)\right)$ in dimensions $n-1$ and $n$ respectively, for any coefficients $K$, and the Hirsch coboundary operator $\partial$ may be expressed in precisely the same terms as in the construction above.

Eilenberg-MacLane note, in their construction of their group $E^{n}(k, G)$, that the associated exact sequence, analogous to the sequence $\left({ }^{*}\right)$ above, is independent of the choice of cocycle $k$ in the characteristic class $k$. This may also be verified for the exact sequence $(*)$ associated with the group $I^{n}\left(C^{*}(B ; K) \otimes_{K} H^{*}(\pi, n ; K)\right)$ or more generally for the sequence associated with $H^{n}\left(\operatorname{Hom}\left(C_{*}(B), I^{*}(\pi, n ; K)\right)\right.$. The required argument is the same as that of Eilenberg-MacLane. However, it is possible to prove that the exact sequence is independent of the choice of cocycle in the characteristic class, by an alternative and more general method which may be of interest. I shall end this section by indicating this method.

First, then, it is evident that the groups $H^{n+1}\left(C^{*}(B ; K) \otimes_{K^{*}} H^{*}(\pi, n ; K)\right)$, for $n>1$, and $H^{n+2}\left(C^{*}(B ; K) \otimes_{K} H^{*}(\pi, n ; K)\right)$, for $n>2$, can, in theory be made explicit by the above methods. One requires, of course, the cohomological operations associated respectively with the groups $H^{n+1}(\pi, n ; K)$ and $H^{n+2}(\pi, n+2 ; K)$, i.e. the "Bochstein coboundary operators" associated with elements of Ext Abel $(\pi, K)$, and the "Steenrod Squares" associated with the group of trace functions from $\pi$ to $K$. 
In these terms results corresponding to Theorems 2 and 3 of [4] can be reobtained. The required arguments are analogous to, and generalize, the arguments giving rise to the exact sequence $\left(^{*}\right)$ in the case of

$$
H^{n}\left(C^{*}(B ; K) \otimes_{K} H^{*}(\pi, n ; K)\right),
$$

considered above. Thus for example in dimension $(n+1), n>1$, the relevant cochain group is

$$
C^{n+1}(B ; K)+C^{1}(B ; K) \otimes_{K} \operatorname{Hom}(\pi, K)+\operatorname{Ext} \operatorname{Abel}(\pi, K),
$$

and if $\delta_{\theta}: H^{n+1}(B ; \pi) \rightarrow H^{n+2}(B ; K)$ is the "Bochstein coboundary operator" corresponding to the extension $\theta \in \operatorname{Ext} \operatorname{Abel}(\pi, K)$, and $\delta_{\theta} k^{n+1}$ any cocycle in $\delta_{\theta} k^{n+1}$, then the Hirsch coboundary operator may be defined in dimension $(n+1)$ by setting

$$
\partial\left(b^{n+1}, b^{1} \otimes \rho, \phi\right)=\left(\partial_{B} b^{n+1}+(-1)^{n+1} \rho k^{n+1} \cup b^{1}-\delta_{\phi} k^{n+1}, \partial_{B} b^{1} \otimes \rho, 0,0\right)
$$

for all triples $\left(b^{n+1}, b^{1} \otimes \rho, \phi\right)$, where $b^{n+1} \in C^{n+1}(B ; K), b^{1} \in C^{1}(B ; K)$, $\rho \in \operatorname{Hom}(\pi, K)$, and $\phi$ is any basis element of the $K$-module Ext Abel $(\pi, K)$.

Taking $H^{1}(B ; K)=0$ as in [4], it may be verified that the resulting cohomology group, $H^{n+1}\left(C^{*}(B ; K) \otimes_{K} H^{*}(\pi, n ; K)\right)$, has associated with it the exact sequence

$\left.{ }^{* *}\right) \quad 0 \rightarrow \mathfrak{S}^{n+1}(B ; K) \rightarrow H^{n+1}\left(C^{*}(B ; K) \otimes_{K} H^{*}(\pi, n ; K)\right) \rightarrow \operatorname{Ext~Abel}{ }^{*}(\pi, K) \rightarrow 0$

where $\operatorname{Ext} \operatorname{Abel}^{*}(\pi, K)$ is the subgroup of Ext Abel $(\pi, K)$ which consists of those extensions $\theta$ such that $\delta_{\theta} k^{n+1}=0$, and $\mathfrak{S}^{n+1}(B ; K)$ denotes the factor group of $H^{n+1}(B ; K)$ by the subgroup of elements of the form $\rho^{*} k^{n+1}$, for all $\rho \in \operatorname{Hom}(\pi, K)$.

This last verification may be carried out relatively easily; but in higher dimensions the calculation of these group extension properties enjoyed by $H^{n+r}\left(C^{*}(B ; K) \otimes_{K} H^{*}(\pi, n ; K)\right.$ ), (even for example the composition serics property in dimension $(n+3)$ corresponding to Theorem 3 of [4]), proves more tractable using standard spectral sequence methods. It is moreover these methods which prove that the various group extensions and composition series given for the groups $I I^{n+r}\left(C^{*}(B ; K) \otimes_{K} I^{*}(\pi, n ; K)\right)$ are independent of the various choices of cocycles in the classes $T(y) k^{n+1}$.

In fact these various group extension, and, more generally, composition series, properties enjoyed by $I^{*}\left(C^{*}(B ; K) \otimes_{K} I^{*}(\pi, n ; K)\right)$ in this context follow immediately from an examination of the termination of the spectral sequence associated with $C^{*}(B ; K) \otimes_{K} H^{*}(\pi, n ; K)$, subject to the differential $\partial$ of the Hirsch method, and filtered as in $\$ 2$ by setting

$$
F_{i}\left(C^{*}(B ; K) \otimes_{K} H^{*}(\pi, n ; K)\right)=\sum_{r \geqq i} C^{r}(B ; K) \otimes_{K} H^{*}(\pi, n ; K) .
$$


This sequence terminates, of course, in the graded group associated with the cohomology group $H^{*}\left(C^{*}(B ; K) \otimes_{K} H^{*}(\pi, n ; K)\right)$ suitably filtered, and the required examination of this group is entirely analogous to that carried out in [4] on the spectral sequence associated with $(E, B, F)$, in its relationship to $H^{*}(E, K)$. I shall not repeat these arguments.

Thus, as is usual when spectral sequences are employed, the various group extension and composition series properties enjoyed by

$$
H^{*}\left(C^{*}(B ; K) \otimes_{K} H^{*}(\pi, n ; K)\right)
$$

in dimensions $<2 n$ are precisely summed up in the statement that the termination of the spectral sequence associated with $C^{*}(B ; K) \otimes_{K} H^{*}(\pi, n ; K)$ is the graded group associated with $H^{*}\left(C^{*}(B ; K) \otimes_{K} H^{*}(\pi, n ; K)\right)$ suitably filtered.

Now the arguments employed in $\$ 2$ imply that the monomorphism

$$
u=C^{*}(B ; K) \otimes_{K} H^{*}(\pi, n, K) \rightarrow C^{*}(E ; K)
$$

induces an isomorphism of the spectral sequences associated with $C^{*}(B ; K)$ $\otimes_{K} H^{*}(\pi, n ; K)$, and with $(E, B, F)$. Thus since different choices of the cocycles $T(\boldsymbol{y}) k^{n+1}$ in the cohomology classes $T(\boldsymbol{y}) \boldsymbol{k}^{n+1}$ cannot alter the spectral sequence associated with $(E, B, F)$, we have the following general result which, of course, contains the fact that the sequence $\left({ }^{*}\right)$ is independent of the choice of the cocycle $k^{n+1}$ in the class $k^{n+1}$, and that the sequence $\left({ }^{* *}\right)$ is independent of the choice of $\delta_{\theta} k^{n+1}$ in $\delta_{\theta} k^{n+1}$.

The termination of the spectral sequence associated with $C^{*}(B ; K)$ $\otimes_{K} H^{*}(\pi, n ; K)$, i.e. the graded group associated with

$$
H^{*}\left(C^{*}(B ; K) \otimes_{K} H^{*}(\pi, n ; K)\right)
$$

suitably filtered, as independent of the choice of cocycles $T(y) k^{n+1}$ in the cohomology classes $T(y) k^{n+1}$ occurring in the definition of the Hirsch coboundary operator in $C^{*}(B ; K) \otimes_{K} H^{*}(\pi, n ; K)$.

As usual, if necessary, $C^{*}(B ; K) \otimes_{K} H^{*}(\pi, n ; K)$ can be replaced by $\operatorname{Hom}\left(C_{*}(B), I I^{*}(\pi, n ; K)\right)$ in the result. The argument is unaltered.

5. Comparison of the Hirsch method with the spectral sequence method. It would seem worthwhile to end by comparing, briefly, the value of the Hirsch method with that of the method of spectral sequences, in connection with the practical problem of computing the cohomology of fibre spaces $(E, B, F)$ with fibre of type $\mathfrak{K}(\pi, n)$.

As a matter of practice the coefficients which appear to be of interest, in respect of spaces of type $\kappa(\pi, n)$, are the integers $Z$ and the fields $Z_{p}$, of integers modulo a prime $p$. Since the groups $I^{m}(\pi, n ; Z)$ will always have torsion for infinitely many values of $m$, one is left only with fields of coefficients in any practical application of the Hirsch method to fibre spaces with fibre of type $\mathfrak{K}(\pi, n)$. 
Thus the problem reduces to calculating the ranks of vector spaces $H^{m}\left(E ; Z_{p}\right)$. In practice then, there seems little to choose between the method of Hirsch as presented here and that of spectral sequences $\left({ }^{14}\right)$. For in computing the rank of $H^{m}\left(C^{*}\left(B ; Z_{p}\right) \otimes_{z_{p}} H^{*}\left(\pi, n ; Z_{p}\right)\right)$, as constructed in $\S 3$ above, for $0<m<2 n$, the most reasonable practical way to proceed seems to be to consider, as in $\$ 4$ above, the spectral sequence associated with $C^{*}\left(B ; Z_{p}\right)$ $\otimes_{z_{p}} H^{*}\left(\pi, n ; Z_{p}\right)$, (with Hirsch coboundary operator $\partial$ ), and to compute the $E_{\infty}$ terms of this spectral sequence. For, since a field of coefficients is taken, all group extensions are trivial, and

$$
\operatorname{Rank}\left\{H^{m}\left(C^{*}\left(B ; Z_{p}\right) \otimes_{z_{p}} H^{*}\left(\pi, n ; Z_{p}\right)\right)\right\}=\sum_{r+s=m} \operatorname{Rank}\left\{E_{\infty}^{r, s}\right\} .
$$

But, again as noted in $\S 4$, the spectral sequence associated with $(E, B, F)$ is isomorphic at the $E_{\infty}$ stage with the sequence associated with $C^{*}\left(B ; Z_{p}\right)$ $\otimes_{z_{p}} H^{*}\left(\pi, n ; Z_{p}\right)$. Thus one could equally well start with $(E, B, F)$ and proceed directly to the examination of its spectral sequence.

In any such examination of course, one requires at least a complete knowledge of the transgression. This is given here, for the results of $\mathrm{H}$. Cartan [2] show that, at least when $\pi$ is finitely generated, one can take as a system of generators of the algebra $H^{*}\left(\pi, n ; Z_{p}\right)$ suitably iterated Steenrod powers of the fundamental class of $H^{*}\left(\pi, n ; Z_{p}\right)$ and of the "Bochstein coboundary" of the fundamental class. The Steenrod and Bochstein operations commute with the transgression, so that the transgression is expressible in terms of the iterated Steenrod powers of the characteristic class of the fibre space and of its "Bochstein coboundary." The procedure would generalize that employed in [4] where only the lower dimensional operations in $H^{*}(\pi, n ; K)$ appeared, but would employ precisely the same spectral sequence arguments.

The difficulties met in either spectral sequence are essentially those of computing the coboundary operators of the spectral sequence on the "base terms" $E_{r}^{p, 0}$, and of computing the rank of a subgroup, of the "fibre term" $E_{2}^{0, Q}$, which is generated by elements corresponding to cohomology operations which annihilate the characteristic class of the fibre space.

On the other hand, as noted in $\$ 4$, the Hirsch method does have the advantage, over the methods of [4], that it leads to fairly simple generalizations, at least in dimensions $n+1, n+2$, of the Eilenberg-MacLane group $E^{n}(k, G)$. These generalizations are definable in a purely algebraic manner and may

$\left.{ }^{14}\right)$ (Added August 22, 1958) This is, of course, not to say that there is no convenient method of, for example, combining the two methods to the advantage of the Hirsch method. Thus Professor Hirsch has recently pointed out to me that by choosing a suitable filtration of the group $H^{*}(F ; K)$ one can in certain circumstances (including the case when $F$ is of type $\mathcal{K}(\pi, n)$ ) make the computations involved in the use of his method very much more rapidly and conveniently, by spectral sequence methods. 
prove of interest in connection with suitable algebraic $n$-types, just as, of course, $E^{2}(k, G)$ is of interest in connection with the algebraic 3-type of a two dimensional complex $\left({ }^{15}\right)$. I hope to consider these groups elsewhere, in this connection.

\section{BIBLIOGRAPHY}

1. A. Borel, Sur la cohomologie des espaces fibrés principaux et des espaces homogenes de groupes de Lie compacts, Ann. of Math. vol. 5 (1953) pp. 115-207.

2. H. Cartan, Seminaire H. Cartan de l'Ecole Normale Superieure, 1954-1955, Paris (mimeographed).

3. H. Cartan and S. Eilenberg, Homological algebra, Princeton, 1956.

4. W. H. Cockcroft, The cohomology groups of a fibre space with fibre a space of type $\mathcal{K}(\pi, n)$, Proc. Amer. Math. Soc. vol. 7 (1956) pp. 1120-1126.

5. S. Eilenberg and Saunders MacLane, Relations between homology and homotopy groups of spaces II, Ann. of Math. vol. 51 (1950) pp. 514-533.

6. - Acyclic models, Amer. J. Math. vol. 75 (1953) pp. 189-199.

7. G. Hirsch, Sur les groupes d'homologie des espaces fibres, Bull. Soc. Math. de Belgique vol. 6 (1953) pp. 79-96.

8. Saunders MacLane, Cohomology theory in abstract groups. III, Ann. of Math. vol. 50 (1949) pp. 736-761.

9. J. P. Serre, Homologie singulière des espaces fibrés. Applications, Ann. of Math. vol. 54 (1951) pp. $425-505$.

UNIVERSITY OF SOUTHAMPTON, SOUTHAMPTON, ENGLAND

(15) See e.g. [8]. 